\section{Marihuana schadet der Lunge doch}

Tan WC et al. The effects of marijuana smoking on lung function in older people. Eur Respir ] 2019; doi:10.1183/13993003.00826-2019

Die schädlichen Folgen des Nikotinkonsums sind gut erforscht und unbestritten. Weniger untersucht und kontrovers diskutiert sind hingegen die Auswirkungen einer langjährigen Marihuana-Exposition. Die Studien COLD und CanCOLD liefern nun Daten zur Lungenschädigung. Mit einem Nachteil: Die meisten Konsumenten rauchten auch Tabak.

Mit Ausnahme der aktiven Substanzen Delta-9-Tetrahydrocannabinol und weiterer Cannabinoide bestehen qualitativ große Ähnlichkeiten zwischen Marihuana und Tabak. Die meisten epidemiologischen Studien wiesen auf eine Assoziation von chronischen respiratorischen Symptomen und Marihuana-Konsum hin. Der Effekt auf die Lungenfunktion blieb aber offen. Die COLD-Studie (Canadian Obstruktive Lung Disease Study mit 5291 Personen $\geq 40$ Jahre ergab die Häufigkeit einer chronisch obstruktiven Lungenerkrankung (COPD) bei Konsumenten von Marihuana (M), Tabak (T), Marihuana und Tabak (MT) sowie bei Personen, die beide Substanzen nie geraucht hatten (NS). Als Raucher galten Teilnehmer, die $\geq 365$ und/oder $\geq 50$ Joints konsumiert hatten. Ein starker Abusus bestand bei >20 Joint- oder Packungs-Jahren. JointJahre ergaben sich aus dem Produkt tägliche Joints $\times$ Konsum-Jahre. Die CanCOLDStudie enthielt ein Subset von $1285 \mathrm{~Pa}$ tienten mit einer COPD $\left(\mathrm{FEV}_{1} / \mathrm{FVC}<0,7\right)$, die jede 18 Monate eine Spirometrie erhielten, um die Abnahme der Lungenfunktion in Abhängigkeit vom Raucherstatus zu untersuchen.

Die Verteilung der Rauchertypen war in den Studien vergleichbar. Ein ausschließlicher Marihuana-Konsum war selten (COLD 17\% und CanCOLD 14\%). $83 \%$ und $86 \%$ rauchten sowohl Tabak als auch

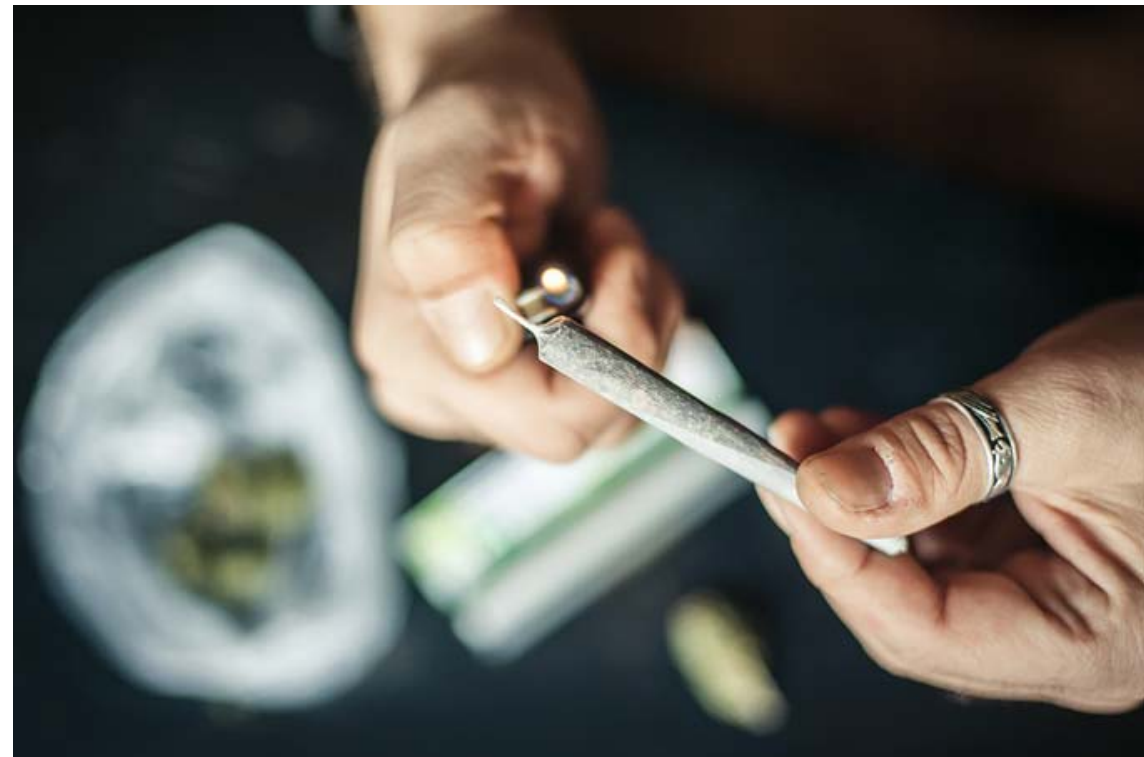

Marihuana ist nicht nur eine Jugenddroge. In der USA konsumieren viele ältere Menschen neben Tabak auch regelmäßig Cannabinoide. Bildquelle: guruXOX - stock.adobe.com

Marihuana. Zudem bestand in der MTGruppe ein deutlich stärkerer Konsum. Die kumulative Exposition betrug in COLD 17,02 Joint-Jahre (MT) vs. 7,23 (M). Der Unterschied war in CanCOLD mit 16,68 vs. 5,45 Joint-Jahren noch ausgeprägter. Unter Berücksichtigung der Nikotinexposition war ein MarihuanaKonsum mit der Wahrscheinlichkeit für eine COPD assoziiert:

- 1 - 5 Joint-Jahre OR 1,39

(95\%-KI 0,96-2,02)

- >5-20 Joint-Jahre OR 1,28

(95\%-KI 0,84-1,95) und

- >20 joint-Jahre OR 2,45

(95\%-KI 1,55-3,88).

Für starke Marihuana-Konsumenten war die Risikosteigerung signifikant. Die schädlichen Konsequenzen von Marihuana zeigten sich auch in einer stärkeren Abnahme der Lungenfunktion. Im Vergleich zu Personen, die nie geraucht hatten, nahm die $\mathrm{FEV}_{1}$ bei starken Tabakrauchern (21,1 ml/Jahr) und bei starken Marihuana-Rauchern $(29,6 \mathrm{ml} / \mathrm{Jahr})$ deutlicher ab. Sowohl in der M- als auch in der T-Gruppe profitierten die Teilnehmer von einem Rauchstopp, der die pulmonale Verschlechterung verlangsamte.
FAZIT

Ein Marihuana-Konsum amplifiziert die schädlichen Effekte des Nikotinkonsums, belegt am gesteigerten Risiko für eine COPD und die $\mathrm{FEV}_{1}$-Abnahme, meinen die Autoren. Frühere Untersuchungen hatten teilweise nur marginale Auswirkungen gezeigt. Tan et al. führen dies u. a. auf heterogene Studiendesigns und vor allem jüngere Teilnehmer zurück. Die signifikante Assoziation von kumulativen JointJahren und COPD spräche für die nikotinunabhängige Schädigung durch Marihuana. Es bestehe ein dringender Bedarf an größeren Langzeitstudien. Die Annahme, Marihuana sei eine Jugenddroge ist falsch: In den USA seien Cannabinoide nach Tabak die häufigsten von Älteren konsumierten illegalen Substanzen.

Dr. med. Susanne Krome, Melle 